González Lorente, Cristina; Martínez Clares, Pilar (2016). Expectativas de futuro laboral del universitario de hoy: un estudio internacional. Revista de Investigación Educativa, 34(1), 167-183.

DOI: http://dx.doi.org/10.6018/rie.34.1.232071

\title{
Expectativas de futuro laboral del universitario de hoy: un estudio internacional
}

\section{Expectations of Getting a Job as aUniversity Student Today: An International Study}

\author{
Cristina González Lorente \\ Pilar Martínez Clares \\ Departamento de Métodos de Investigación y Diagnóstico en Educación \\ Facultad de Educación. Universidad de Murcia
}

\begin{abstract}
Resumen
Ante las elevadas tasas de desempleo juvenil crece la preocupación y el interés por alcanzar un mejor ajuste entre las competencias profesionales y los requerimientos del mercado de trabajo. La educación tiene un compromiso, el bienestar de la sociedad, y como tal, el proceso de inserción socio-laboral se convierte en una parte esencial de la misma. Este trabajo desde una perspectiva internacional, la Universidad de Murcia y de la Universidad Católica de Córdoba (Argentina), trata de analizar cuáles son los factores que pueden influir en las percepciones y creencias del mercado laboral, desde la visión del estudiante de último curso de grado y las posibilidades factibles de acceder al mismo una vez finalizada la formación inicial, a través de un estudio no experimental, exploratorio y transversal, tipo encuesta. Los resultados son útiles para avanzar en el desarrollo, mejora y funcionalidad de la Educación Superior.
\end{abstract}

Palabras clave: Educación Superior; empleo; orientación; inserción socio-laboral.

\begin{abstract}
Considering the higher rates of youth unemployment, the demand and interest for achieving a better adjustment between the professional competences and the requirements of the job mar-
\end{abstract}

Correspondencia: c.gonzalezlorente@um.es ; pmclares@um.es 
ket have increased. One of the most important goals of education, and in turn the well-being of society, is entering the workforce. From the international perspectives at the University of Murcia (UM) and the Catholic University of Córdoba (UCC), this paper aims to analyze the main factors in the perceptions and beliefs about labor market. In addition, it is focused on the final year students' views and opinions about the real possibilities of entering the labor force after completing their initial training at university. An experimental, exploratory and transversal study with a survey was applied. The results are useful to make progress in the development, improvement and functionality of Higher Education.

Keywords: Higher Education;employment; guidance; entering to workforce.

\section{Introducción}

Las consolidadas relaciones en el mundo del trabajo del pasado son en el presente efímeras e inestables, generando una ruptura con la forma en la que tradicionalmente los estudiantes se acercaban al mercado laboral. Si hasta hace poco tiempo los jóvenes con formación universitaria se incorporaban de manera casi natural y fluida al mercado de trabajo, en la actualidad se ven inmersos en complejas y múltiples formas de transición a la vida activa.

La transición se entiende como un proceso de cambio (Jurado de los Santos, Olmos Rueda y Pérez Romero, 2015; Martínez Clares, 2008) donde se relacionan los determinantes personales (historia personal y profesional) con los determinantes contextuales o situacionales para que de manera recíproca, la persona pueda construir su identidad y aprender nuevos roles en "la tendencia hacia la autorrealización" (Martínez Clares, 2008 , p. 260). Por esta razón, desarrollar la carrera profesional únicamente en base a la formación inicial es ya una realidad obsoleta, dada la rápida caducidad y volatilidad que lleva implícita la formación en una sociedad del conocimiento. La tecnología avanza a más velocidad y modifica la forma en la que dicha formación se transmite con nuevas modalidades de enseñanza como e-learning, mooc, entornos virtuales de aprendizaje, etc. (Centeno Moreno y Cubo Delgado, 2013), haciéndola más accesible y rápida.

Sumidos en la modernidad líquida (Bauman, 2008) con los continuos cambios que en ella acontecen, la formación y el aprendizaje a lo largo de la vida se hacen imprescindibles, incluso para aquellos que poseen una titulación universitaria si quieren mantenerse en el actual mercado laboral. De hecho, estos titulados universitarios dejan de suponer de facto la credencial que garantiza el conocimiento (Vázquez García, 2015) y los títulos pasan de ser entendidos como billete de destino a formar parte de un pasaporte de salida que necesitan completar y actualizar de manera continua.

Estudios nacionales e internacionales centrados en analizar las causas, consecuencias y efectos del desempleo juvenil (Blossfeld y Mills, 2010; de la Hoz, Quejada y Yánez, 2012; García-Montalvo y Peiró, 2012; Homs, 2014; Matsaganis, Rabemiafara y Ward, 2013) evidencian como cada vez es más difícil obtener dicho "pasaporte", especialmente, entre la población más joven y subrayan la imposibilidad en muchas ocasiones de satisfacer las necesidades personales, sociales y profesionales que junto con la incapacidad de generar ingresos dificulta la proyección laboral futura de esta población.

Ante las elevadas tasas de desempleo juvenil y los índices de sobrecualificación que presentan países como España, el interrogante se sitúa en torno al mercado productivo 
de cada país y a su posibilidad real para absorber esta generalización de estudiantes de Educación Superior. Resulta fundamental conocer qué es lo que las empresas requieren de los titulados universitarios, el grado de preparación con el que estos se incorporan a la actividad empresarial y los aspectos formativos que aquellas consideran mejorables.

Desde esta panorámica es interesante profundizar también en la percepción de quienes experimentan en primera persona el difícil proceso de inserción, es decir, los propios universitarios. Conocer y contrastar dichos perfiles, así como los diferentes factores o variables que pueden influir en sus percepciones del mercado laboral y posibilidades factibles de acceder al mismo una vez finalizada su formación inicial, debe ser prioritario para avanzar en el desarrollo, mejora y funcionalidad de la Educación Superior.

En el escenario internacional (Dumitriu, Timofti y Dumitriu, 2014; Hager y Holland, 2006; Jurado de los Santos et al., 2015; Watts, 2006) se señala como el éxito de los estudiantes a la hora de acceder a un empleo no sólo depende de la formación recibida sino también, de sus circunstancias y actitudes, así como del contexto laboral, social y económico en el que se encuentran, del estado del mercado de trabajo y de las formas en las que opera a nivel local, nacional e internacional. Todos estos factores, externos e internos a la persona, hacen de la inserción socio-laboral un proceso complejo, poliédrico, multidisciplinar y multiprofesional de transición, marcado por la incertidumbre y una serie de problemáticas acentuadas por los constantes cambios tecnológicos, socioeconómicos y culturales, tal y como se representa en la Figura 1.

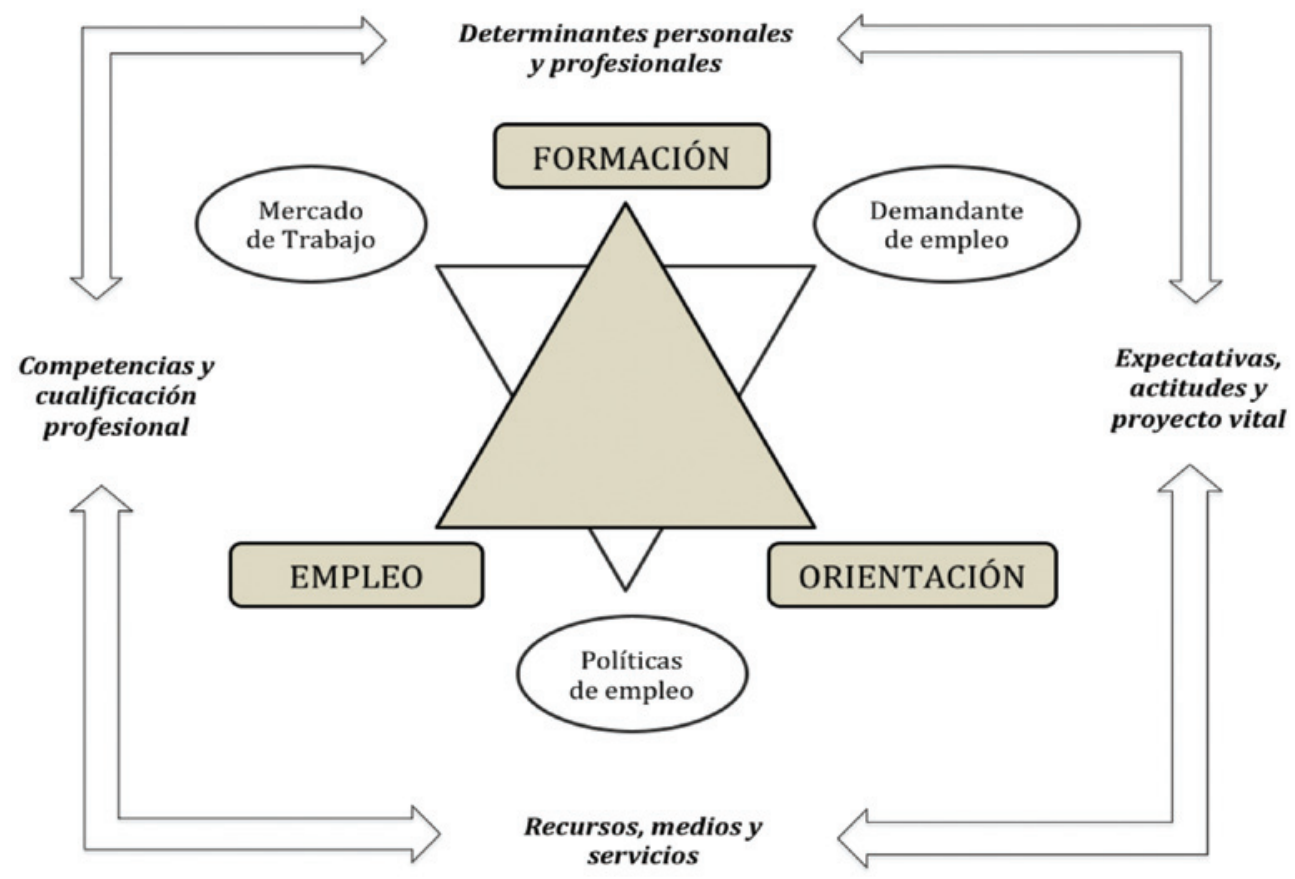

Figura 1. Factores que inciden en el proceso de inserción laboral en función del triángulo básico formación-orientación-empleo. 
El proceso de inserción socio-laboral dependerá en gran medida, de una buena formación de base que permita a las personas adaptarse a los requerimientos sociales y profesionales que demanda el mercado de trabajo (Isus, 2008), pero no de forma exclusiva. Requiere a su vez, de un proceso de orientación y asesoramiento que facilite su proceso de toma de decisiones contrastada, acorde a las alternativas posibles y deseables, tanto a nivel interno como externo, para acceder a un empleo pleno y mantener activa su empleabilidad y ocupabilidad. La Orientación Profesional se convierte así, en eje vertebrador entre el empleo y la formación necesaria para ejercerlo.

Con la intención de profundizar en estos y otros factores que repercuten en el proceso de inserción socio-laboral del universitario, se realiza este estudio desde una perspectiva internacional con estudiantes de dos universidades localizadas en países y realidades diferentes entre sí como son, la Universidad de Murcia (UM, en España) y la Universidad Católica de Córdoba (UCC, en Argentina). Dicho trabajo forma parte de una investigación más amplia desarrollada en ambas universidades con el fin de conocer el proceso para la inserción socio-laboral de los estudiantes y las percepciones y creencias que tienen los estudiantes en sus proyecciones laborales de futuro. Desde este planteamiento general, en este trabajo se plantea un doble objetivo:

1. Conocer y analizar la percepción y valoración que tienen los estudiantes de la UM y UCC sobre sus probabilidades de encontrar empleo a corto plazo.

2. Identificar posibles factores y determinantes que influyen en las creencias que tienen estos estudiantes sobre su inserción socio-laboral.

\section{Método}

\section{Enfoque metodológico y diseño de la investigación}

Para este estudio en particular, se opta por un enfoque metodológico mixto o socio-crítico que permita triangular los datos obtenidos y establecer conclusiones más certeras que ayuden a la comprensión del proceso analizado y al establecimiento de prospectivas.

Para el desarrollo de este estudio se trabaja desde un diseño de investigación no experimental, exploratorio y transversal, tipo encuesta o survey, mediante un cuestionario elaborado ad hoc.

\section{Participantes}

Los participantes de este estudio pertenecen a dos universidades diferentes, de forma que se hace imprescindible conocer las características que le son propias a cada una de ellas para contextualizar los resultados que se obtienen en este trabajo (Tabla 1).

De la Tabla 1 se infiere que existen diferencias notables en cuanto a población, tamaño y financiación, por lo que su tratamiento y comparación a lo largo del mismo, se hacen de forma proporcional al número de participantes en cada una de estas universidades, al tratarse de dos realidades diferentes. 
Tabla 1

Principales indicadores de la estructura interna de la UM y la UCC

\begin{tabular}{lcc}
\hline & $\begin{array}{c}\text { Universidad de } \\
\text { Murcia (UM) }\end{array}$ & $\begin{array}{c}\text { Universidad } \\
\text { Católica de Córdoba } \\
\text { (UCC) }\end{array}$ \\
\hline Tipo de Universidad & Pública & Privada \\
Total de estudiantes de enseñanzas oficiales & 34.404 & 9.810 \\
Titulaciones de Grado & 56 & 35 \\
Total de titulados (egresados) & 5.412 & $1.040^{\underline{a}}$ \\
Total de docentes & 2.460 & 1.575 \\
\hline
\end{tabular}

Nota: a. Dato disponible en la última actualización del INDEC (curso 2011).

Fuente: Universidad de Murcia y Universidad Católica de Córdoba.

Los participantes de este estudio son un total de 155 estudiantes distribuidos de forma proporcional, en 99 estudiantes en la UM y 56 en la UCC. Se encuentran en su último curso de la formación inicial universitaria y pertenecen a la Facultad de Educación de ambas universidades, más la Facultad de Trabajo Social en el caso de la Universidad de Murcia. La elección de estas facultades y sus respectivas titulaciones responde a un criterio de contacto directo del equipo de investigación con las mismas a partir de una estancia de investigación en Córdoba (Argentina).

La distribución de estos participantes, en cuanto a sexo y edad, responde a un $72.7 \%$ y $80.4 \%$ de mujeres en la UM y UCC, respectivamente, siendo en este caso los estudiantes de la UM, más jóvenes respecto a los estudiantes de la UCC (Tabla 2).

Tabla 2

Media de edad de los estudiantes

\begin{tabular}{ccc}
\hline & Media $(\overline{\mathbf{X}})$ & Desv. típica $(\sigma)$ \\
\hline UM & 24.72 & 5.67 \\
UCC & 32.33 & 9.43 \\
\hline
\end{tabular}

\section{Instrumento}

Para la recogida de información se elabora un cuestionario ad hoc, denominado Cuestionario de Orientación e Inserción Laboral (COIL), cuya estructura se organiza en cuatro bloques de estudio recogidos en la Tabla 3.

En base al enfoque metodológico que caracteriza este trabajo, cada uno de estos bloques incluye tanto preguntas cerradas con diferentes tipos de respuesta, como preguntas semicerradas y abiertas que permitan al estudiante justificar sus valoraciones y creencias. 
Tabla 3

Estructura del Cuestionario de Orientación e Inserción Laboral

\begin{tabular}{ccl}
\hline Bloque & Áreas & \multicolumn{1}{c}{ Contenido } \\
\hline I & $\begin{array}{c}\text { Situación personal y } \\
\text { profesional }\end{array}$ & $\begin{array}{l}\text { Sexo, edad, situación laboral actual, participación en } \\
\text { programas de prácticas universitarias e idiomas. }\end{array}$ \\
\hline II & Formación recibida en la \\
Universidad & $\begin{array}{l}\text { Opción de volver a elegir una carrera universitaria di- } \\
\text { ferente, grado de satisfacción con la formación recibida, } \\
\text { preparación para afrontar la inserción laboral en función } \\
\text { de su formación universitaria y aspectos del currículum } \\
\text { más importantes para acceder al mercado laboral. }\end{array}$ \\
\hline III & $\begin{array}{c}\text { Actitudes y expectativas } \\
\text { hacia el empleo }\end{array}$ & $\begin{array}{l}\text { Aspectos a tener en cuenta a la hora de elegir un em- } \\
\text { pleo, objetivos profesionales a corto-medio plazo y mo- } \\
\text { vilidad geográfica. }\end{array}$ \\
\hline IV & $\begin{array}{c}\text { Recursos/servicios que } \\
\text { facilitan la inserción } \\
\text { laboral }\end{array}$ & $\begin{array}{l}\text { Información acerca de las salidas profesionales de la ca- } \\
\text { rrera universitaria, métodos utilizados en la búsqueda } \\
\text { de empleo, Formación y Orientación Profesional, insti- } \\
\text { tuciones a las que acuden y satisfacción. }\end{array}$ \\
\hline
\end{tabular}

\section{Procedimiento}

El proceso de investigación seguido pasa por una serie de fases cuya secuencia es la siguiente:

$1^{\mathrm{o}}$. Planteamiento del problema de investigación con una cuestión de partida (pregunta de investigación) ¿cómo experimentan los estudiantes de dos universidades situadas en realidades y países diferentes, su proceso de inserción laboral?; $2^{\circ}$. Revisión de la literatura y concreción de los objetivos de estudio; $3^{\circ}$. Diseño inicial del cuestionario (COIL) como instrumento de recogida de información. Para garantizar su validez, se realiza un proceso de validación de contenido de tipo cualitativo mediante la técnica de juicio de expertos formado por profesionales de la UM y la UCC, tras cuyo análisis de concreción, pertinencia y adecuación, se tienen en cuenta las correcciones dadas en torno a los ítems utilizados y su redacción, para una mayor comprensión por parte del alumnado de ambas universidades; $4^{\circ}$. Recogida de información en el curso académico 2013/2014; 5o. Categorización y análisis de la información y 6o․ Interpretación de resultados y redacción del informe de investigación.

\section{Análisis de los datos}

El análisis de los datos se realiza a través de la combinación de dos programas informáticos de acuerdo al carácter mixto de las respuestas dadas por los estudiantes. La información de carácter cuantitativo se examina mediante el paquete estadístico SPSS v.20 aplicando la estadística descriptiva e inferencial, en este último caso con la prueba no paramétrica de U de Mann-Whitney. Los datos cualitativos se analizan a 
través del programa Atlas.ti v7 bajo el criterio de reducción de información temático y una estrategia inductiva. Con esta reducción se pretende como señalan Sabariego-Puig, Vilà-Baños y Sandín-Estaban (2014), seleccionar, focalizar y abstraer las respuestas de los estudiantes en unidades de significado (códigos y categorías), utilizando el método de comparación constante que permite encontrar similitudes y divergencias entre las respuestas analizadas.

\section{Resultados}

Los primeros resultados se articulan en torno a la pregunta "¿esperas encontrar empleo en un plazo máximo de 6 meses una vez terminados tus estudios?", una cuestión de reflexión con tres opciones de respuesta que incluye un apartado abierto para la justificación argumentada del estudiante y que pueda explicar de manera más concreta y precisa, la razón de dicha percepción o expectativa laboral a corto plazo. En un primer momento, el análisis descriptivo refleja unos resultados bastante dispares respecto a la percepción que tiene el alumnado de la Universidad de Murcia (UM) y la Universidad Católica de Córdoba (UCC) (Figura 2).

$\because \mathrm{UM}=\mathrm{UCC}$

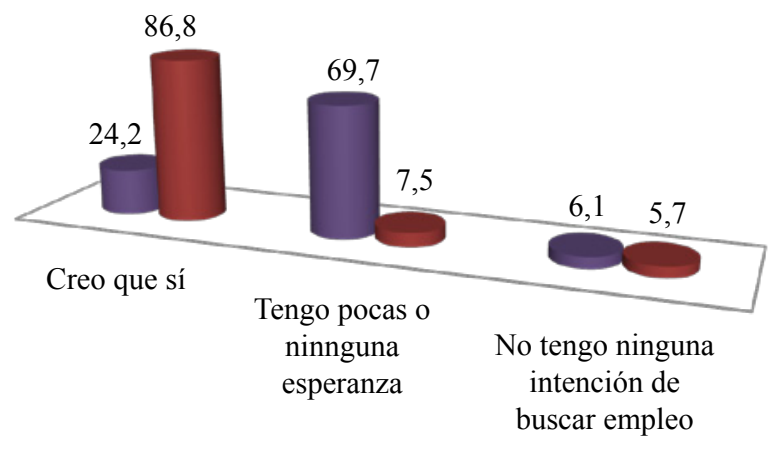

Figura 2. ¿Esperas encontrar empleo en un plazo máximo de 6 meses una vez terminados tus estudios? Distribución de respuestas.

Los estudiantes de la UCC tienen unas expectativas más altas o positivas para encontrar empleo en breve plazo (87\%) mientras que los estudiantes de la UM, tienen una precepción más negativa o desesperanzadora (cerca del 70\%).Unas diferencias que se pueden corroborar a través de la estadística inferencial, tras la aplicación de la prueba U de Mann-Whitney las diferencias significativas $(p=.000)$ encontradas entre las respuestas y/o percepciones de los estudiantes de ambas universidades.

Estas diferencias tan notables entre la percepción de los estudiantes y la clara tendencia en las opciones de respuesta, derivan directamente en el análisis cualitativo de aquellas que obtienen un porcentaje más elevado; "creo que sí" para los estudiantes de la Universidad Católica de Córdoba y "tengo pocas o ninguna esperanza” entre aquellos 
que cursan sus estudios en la Universidad de Murcia, descartándose la opción 3 por disponer de escasas respuestas y argumentos, lo que a su vez permite inferir como realmente los estudiantes encuestados tienen intenciones de buscar un empleo al finalizar sus estudios, con la diferencia de tener más esperanzas de conseguirlo en función de la universidad en la que se obtienen dichos datos.

En este análisis cualitativo, las diferencias en la percepción se pueden combinar de acuerdo a tres grandes categorías: mercado laboral, formación universitaria y actitud del estudiante (Tabla 4) y revelan cierta similitud entre los argumentos vertidos en ambas universidades, si bien las utilizan para justificar percepciones opuestas en muchos casos respecto a las posibilidades de encontrar empleo a corto plazo.

Tabla 4

Codificación y descripción de la información cualitativa

\section{Categoría 1: Mercado laboral}

\begin{tabular}{|c|c|}
\hline Códigos & Descripción \\
\hline $\begin{array}{l}\text { Crisis } \\
\text { económica }\end{array}$ & $\begin{array}{l}\text { La actual crisis financiera y de las economías de mercado, se considera un im- } \\
\text { portante hándicap a la hora de afrontar su proceso de inserción socio-laboral. }\end{array}$ \\
\hline $\begin{array}{l}\text { Contactos } \\
\text { personales }\end{array}$ & $\begin{array}{l}\text { La falta de una red sólida de contactos a los que recurrir en caso de búsqueda } \\
\text { activa de empleo. }\end{array}$ \\
\hline $\begin{array}{l}\text { Titulación } \\
\text { universitaria }\end{array}$ & $\begin{array}{l}\text { Relacionado con las expectativas de promoción social y laboral asociadas tra- } \\
\text { dicionalmente a los estudios universitarios. }\end{array}$ \\
\hline Oferta laboral & $\begin{array}{l}\text { Todo aquello vinculado con las posibilidades reales de la economía y el mer- } \\
\text { cado laboral de cada país, para poder ofrecer puestos de trabajo acordes a la } \\
\text { oferta formativa de su sistema educativo. }\end{array}$ \\
\hline $\begin{array}{l}\text { Experiencia } \\
\text { laboral }\end{array}$ & $\begin{array}{l}\text { Incremento de los requisitos para acceder al mercado laboral, entre los que } \\
\text { se encuentra uno de los más difíciles de cumplir para la población más joven, } \\
\text { esto es, su experiencia laboral previa. }\end{array}$ \\
\hline En activo & $\begin{array}{l}\text { Universitarios que compaginan sus estudios con algún trabajo remunerado, } \\
\text { con las consecuencias directas que representa para el proceso de inserción } \\
\text { laboral tras finalizar la formación en educación superior. }\end{array}$ \\
\hline
\end{tabular}

Categoría 2: Educación Superior

Códigos

Formación

Competencia
Descripción

El desarrollo y relevancia de sus estudios de Educación Superior, así como el hecho de necesitar más formación complementaria y/o específica para encontrar empleo de acuerdo a las preferencias de cada estudiante.

El elevado número de personas que en la actualidad están en posesión de un título de Educación Superior, genera un gran desajuste entre aquellos puestos que requieren de una mayor, o en su caso, menor cualificación, de forma que aumenta exponencialmente la competencia entre los estudiantes. 


\section{Categoría 3: Actitud del estudiante}

\section{Códigos}

Autoconfianza

Vocación

Emigrar

Edad

\section{Descripción}

Mayor o menor confianza del alumnado en función de la universidad analizada acerca de las competencias adquiridas durante la Educación Superior.

Predisposición positiva fundamentalmente de los estudiantes de la UCC hacía los estudios universitarios elegidos y las salidas profesionales de los mismos.

Cuando la única opción para acceder al mercado laboral que se visualiza como posible entre quienes estudian en la UM es la de buscar fuera de las fronteras nacionales.

Limitación que los estudiantes señalan por ser demasiado jóvenes para que un empresario les ofrezca las mismas oportunidades y condiciones laborales que a una persona con mayor edad.

En ambas universidades, predomina la situación actual del mercado laboral como principal eje sobre el cual, los universitarios focalizan la mayoría de sus expectativas; y lo que en unos casos es visto como aspecto potenciador, en otros, parece limitar. En este sentido, los universitarios de la UCC conciben el mercado laboral actual como un escenario "con grandes oportunidades para los titulados universitarios", donde "la oferta laboral es bastante amplia" debido a la actual situación de crecimiento económico de la mayoría de las regiones latinoamericanas. Por ello, no es de extrañar que un porcentaje elevado de estos universitarios afirmen compatibilizar sus estudios con un empleo a tiempo completo, hasta alcanzar un 96,4\% de los estudiantes de la UCC que ya trabajan y algo más del $75 \%$, que lo hacen en un puesto de trabajo relacionado directamente con aquello que estudian, tal y como se recoge en la figura 3.
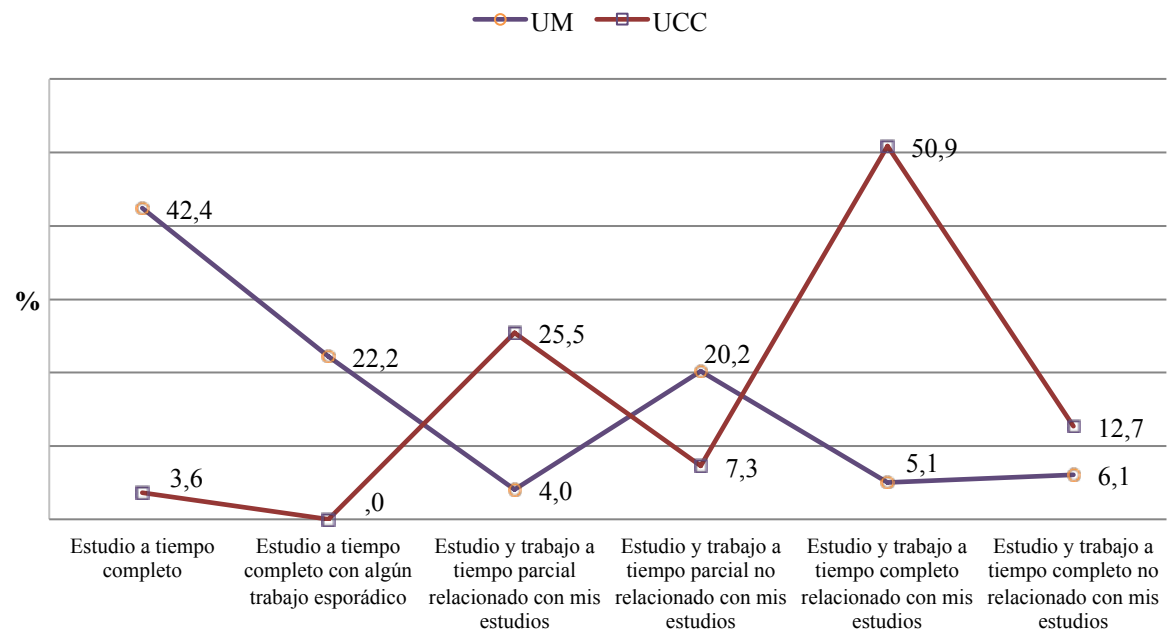

Figura 3. Distribución de los estudiantes en función de su situación laboral actual. 
Por su parte, el perfil del estudiante de la UM es muy diferente y tan sólo un 33.4\% de ellos trabaja al mismo tiempo que continua con sus estudios, dentro de los cuales, un $20.2 \%$ se corresponde con trabajos que no guardan relación alguna con los mismos. Datos que coinciden con las aportaciones de los estudiantes de la UM cuando aluden al actual mercado laboral desde una perspectiva mucho más crítica y desalentadora de la que realiza el estudiante de la UCC.

Muchos de ellos, señalan la "crisis económica" como principal factor exógeno que va a mermar y limitar sus esperanzas de encontrar empleo a corto plazo. En un nivel más concreto, los estudiantes de la UM también aluden a "la escasa contratación actual" en estrecha vinculación con la situación socioeconómica que vive España, o al incremento de personas que disponen de un título de Educación Superior dentro del mercado laboral. En este caso, para los estudiantes de la UM "son muchos los que salen con la misma titulación y muy limitados los puestos vacantes" e incluso en algunos casos llegan a vincular su falta de esperanza con "el poco reconocimiento de su carrera", mientras que los universitarios de la UCC conciben la titulación como un credencial social y laboral realmente muy positivo para acceder a un empleo que "les abrirá nuevas puertas".

Quizás, por esta razón, los estudiantes de la UM encuentran en los programas de prácticas universitarias, una interesante forma de favorecer su empleabilidad y un $54.5 \%$, utilizan esta vía como una buena oportunidad para dar sus primeros pasos hacia su inserción laboral (Figura 3). Por el contrario, aunque los estudiantes de la UCC también coinciden en señalar la utilidad que se esconde detrás de este tipo de programas, un alto porcentaje de dichos estudiantes (59.3\%) no llega a realizarlos, ya que como se veía en la Figura 4, la mayoría de ellos ya se encuentra trabajando.

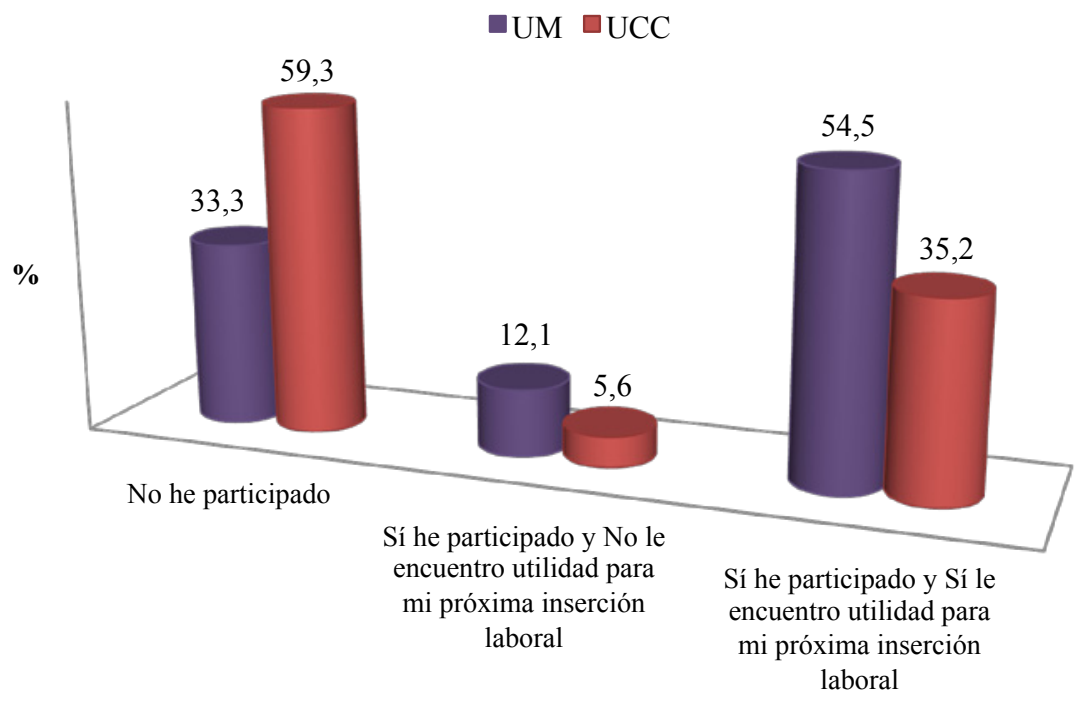

Figura 4. Distribución de los estudiantes en función de su participación en algún programa de prácticas universitario. 
Al igual que ocurre con la forma de entender o concebir el mercado laboral actual, la formación recibida en la universidad UCC se entiende como un elemento clave en su inserción, textualmente expresan que se "sienten capacitados y formados profesionalmente para tal fin" y consideran que su paso por la universidad les proporciona "las herramientas para poder desempeñarse satisfactoriamente" en el ámbito laboral. Los encuestados de la UM creen que no podrán encontrar un empleo a corto plazo porque "les falta formación", afirman que "es escasa" y que necesitan ampliarla con "un posgrado o alguna especialización".

Estas percepciones se pueden contrastar con la valoración cuantitativa dela formación recibida como se aprecia en la Figura 5. Mientras que para los estudiantes de la UM, el nivel de satisfacción con la formación universitaria se sitúa en una ponderación entre "regular" (42.4\%) y "bastante" (45.5\%), la respuesta de los estudiantes de la UCC oscila entre "bastante" (49.1\%) y "mucho" (27.3\%).

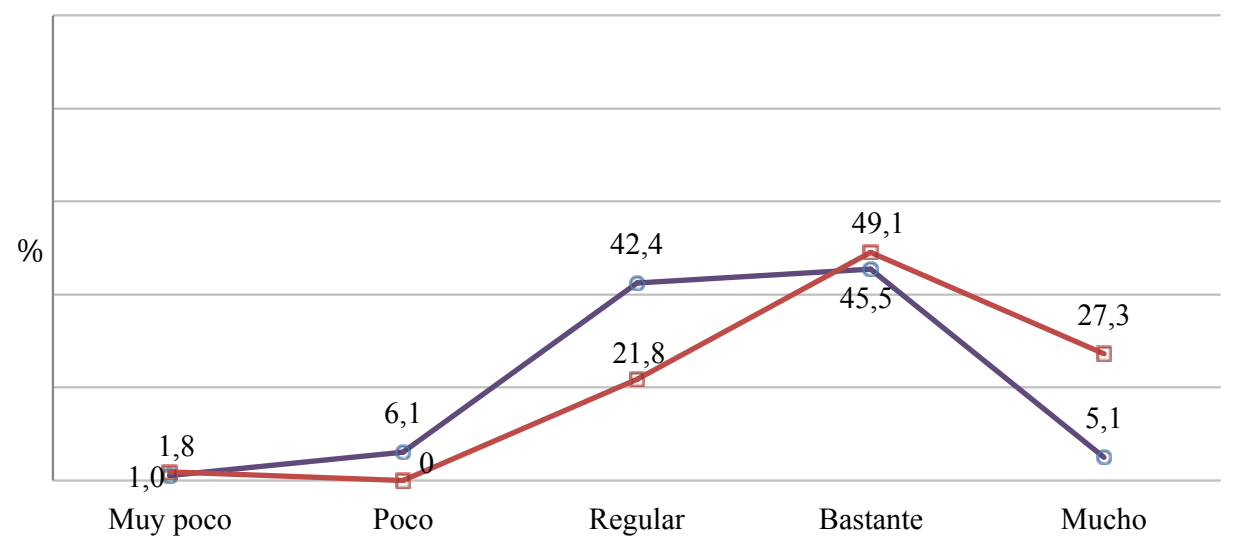

Figura 5. ¿Cuál es el grado de satisfacción en relación a tus estudios universitarios? Distribución de respuestas.

Si bien es cierto que la satisfacción con la formación recibida puede estar condicionada por otros muchos factores, como la situación del mercado laboral de los respectivos países analizada, la Figura 6 muestra como una vez más, los estudiantes de la UCC creen en mayor medida (un $65 \%$ respecto al $22.2 \%$ de quienes estudian en la UM), estar "bastante" o muy preparados para iniciar este proceso de inserción; unos estudiantes que tanto en estos datos como en las respuestas abiertas, presentan de forma general una mejor valoración de todo aquello que su formación universitaria puede aportarles en este proceso.

Por último, es importante destacar como los estudiantes presentan determinadas actitudes que pueden influir en la percepción y motivación a la hora de enfrentarse a su inserción y el valor que el trabajo pueda ocupar entre sus prioridades vitales. En este sentido, aunque en el análisis cualitativo la categoría "Actitud del estudiante" es la que menos citas o referencias ha recabado, no se debe obviar dado el papel fundamental que puede ejercer en la confianza y proyecciones del estudiante. 


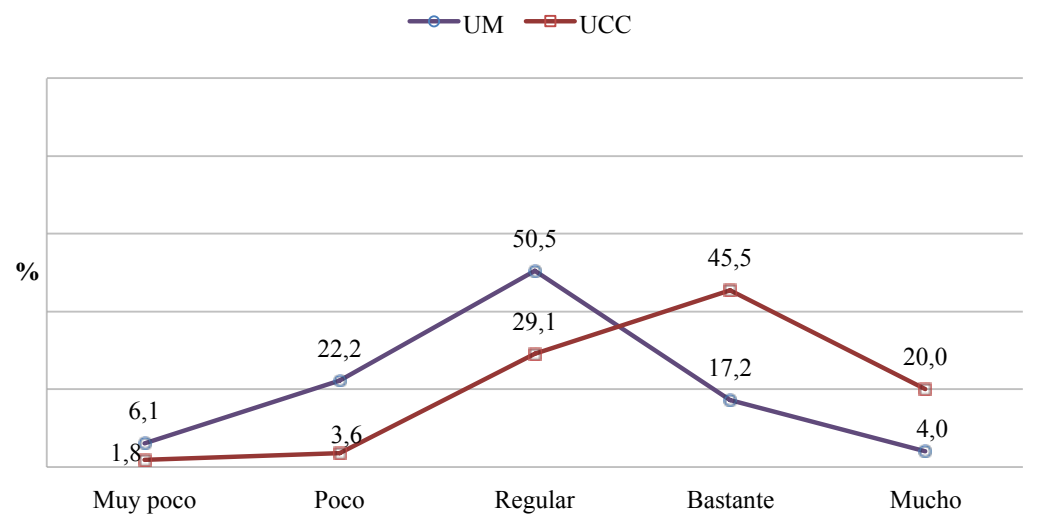

Figura 6. Con la formación recibida en tus estudios universitarios, ¿te sientes preparado para tu próxima IL? Distribución de respuestas.

Es precisamente en esta tercera categoría donde aparecen mayores diferencias en los argumentos, ya que entre el alumnado de la UCC destaca su autoconfianza como persona "altamente cualificada" que ha invertido mucho tiempo y esfuerzo en una formación que consideran de calidad, como señalaban anteriormente y tienen el convencimiento de "poder poner pronto en práctica todo lo aprendido" cuando finalicen sus estudios. Una confianza que está ligada a la vocación y motivación que presentan hacia los estudios superiores elegidos, que en muchas ocasiones les "hace sentir bien y disfrutarlo" así como tener ganas de "trabajar en lo que les gusta" tras años de dedicación a su formación inicial. Visión que contrasta sin duda, con las opiniones de los estudiantes de la Universidad de Murcia, quienes muestran una actitud más pesimista al destacar directamente que "no hay empleo" y que "deben ser realistas con esta situación", ya que su juventud no la consideran un potencial para encontrar un empleo en el mercado laboral actual. Como alternativa, la actitud propuesta es la de "salir fuera del país a aprender otro idioma en profundidad mientras trabajan en lo que puedan", al ser ésta una de las "pocas opciones posibles en la actualidad española".

La flexibilidad y movilidad geográfica es uno de los aspectos más destacados para encontrar un empleo en un mundo cada vez más interconectado donde la estabilidad geográfica, al igual que la estabilidad en un puesto de trabajo, ya no está asegurada. Sin embargo, a pesar de esta imperiosa realidad socio-laboral, ambos colectivos muestran una gran resistencia a la movilidad (Figura 7). Tanto en una Universidad como en otra, los estudiantes manifiestan sus preferencias de permanecer en su contexto más próximo a la hora de buscar empleo y en su caso, a estar dispuestos a ampliar el radio de búsqueda a una distancia no superior a la provincia en la que se encuentran.

Los datos cualitativos analizados en la tercera categoría también coinciden con las propias opciones personales y profesionales de los estudiantes una vez finalizada su carrera universitaria. Buscar cualquier tipo de trabajo como se refleja en la Figura 8, es una de las posibilidades más repetidas entre los estudiantes de la UM. Sin embargo, para los estudiantes de la UCC, buscar cualquier tipo de trabajo representa la última 
de sus intenciones y es la opción de dedicarse a su familia con una media de 3.32 para un máximo de 5 puntos, una de las opciones más repetidas entre este alumnado.

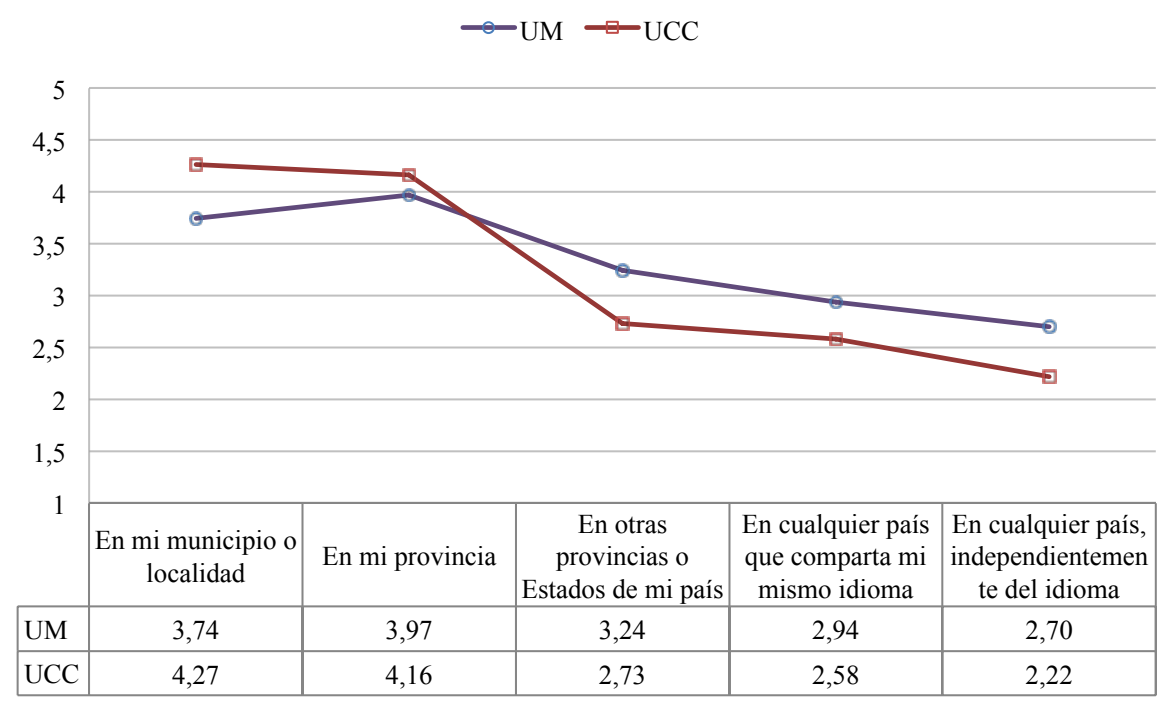

Figura 7. Media de la movilidad laboral de los estudiantes.

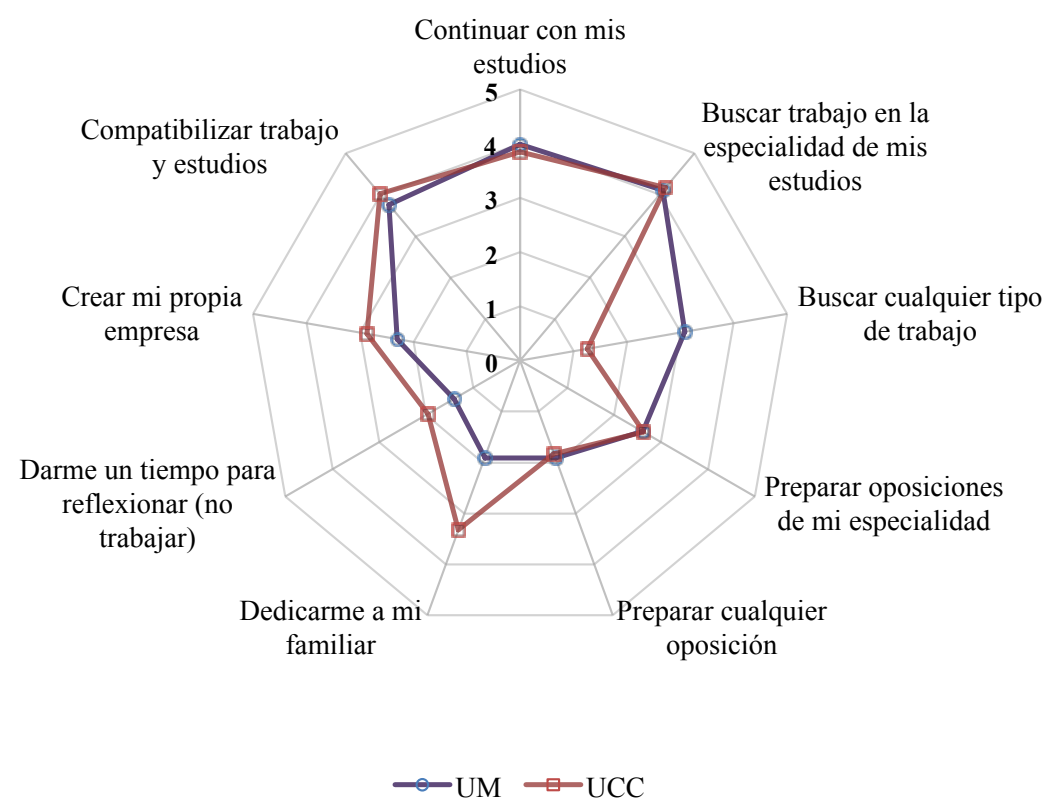

Figura 8. Media de los objetivos e intenciones de los estudiantes una vez finalizada su carrera universitaria. 


\section{Discusión y conclusiones}

Tal y como se refleja a lo largo de este trabajo y coincidiendo con las aportaciones de Echeverría (2008) y Jurado de los Santos et al. (2015), la transición a la vida activa se ve influenciada por diferentes variables personales, sociales, económicas y formativas, que impactan de una manera u otra, tanto en las oportunidades de inserción laboral de los estudiantes, como en la forma en la que este proceso se produce y los actores que intervienen en él.

Entre estos múltiples factores, la edad aparece en este trabajo como un factor que puede situar al estudiante de la UM, en una peor posición para afrontar la inserción laboral por su juventud y falta de experiencia laboral. De hecho, esta es una realidad en la actualidad española, según datos publicados por el Ministerio de Educación Cultura y Deporte de España (MECD, 2013), cada vez son más los universitarios y menor la edad de graduación. Por su parte, otros estudios realizados en Argentina (Brunner, 2012; García de Fanelli y Jacinto, 2010) también sitúan esta media entre los universitarios de este país en torno a los 24 años, coincidiendo cada vez más con las estadísticas de las universidades españolas.

Además de la edad, los participantes señalan la importancia de realizar prácticas durante los estudios universitarios o bien, el hecho de trabajar durante este período; en ambos casos, lo consideran factores coadyuvantes para aumentar las probabilidades de éxito en la inserción socio-laboral, tal y como se corrobora en los estudios realizados por Kessler (2010) o por la Organización Internacional del Trabajo (OIT, 2013).

En el caso concreto de los estudiantes de la UCC, cabe destacar como el mercado laboral al que acceden, aún les permite encontrar trabajos relacionados con su área de estudio pese a las desigualdades sociales y económicas que se viven en Argentina y la segmentación de la Educación Superior (Maurizio, 2011) y son muchos los que compaginan esta actividad con un trabajo a tiempo completo o parcial que en numerosas ocasiones, guarda una estrecha relación con su formación actual. Sin embargo, los estudiantes de la UM parecen obtener esta experiencia de forma más extendida, a través de los programas de prácticas curriculares como indican Escolano et al. (2015) y Zabalza (2011) o bien, a través de prácticas de carácter extracurricular, cada vez más extendidas y demandadas por las empresas.

Quizás, por esta diferencia a la hora de obtener experiencia laboral durante los estudios, la satisfacción con la formación recibida y el valor que le otorgan a la misma también varía en función de la Universidad y su situación laboral. De esta forma, mientras en este estudio se destaca la escasa valoración que los universitarios españoles conceden a su formación inicial en la universidad apoyada por la falta de utilidad que encuentran en los estudios para "lograr una inserción laboral rápida y adecuada" (Alonso, Fernández y Nyssen, 2009, p. 12), los egresados de la UCC y de Argentina en general, se muestran más satisfechos con su formación, dándole un mayor valor a la posibilidad de realizar estudios universitarios como oportunidad de promoción y desarrollo socioeconómico y profesional (J. E. Noriega y Montiel, 2014).

En esta misma línea, se puede inferir que a pesar de los efectos de la crisis económica, los estudiantes de la UM muestran todavía, una gran resistencia para ampliar 
su radio de búsqueda de empleo, de manera que buscarlo en la misma localidad o provincia en la que residen, continua siendo la opción preferida por la mayoría, así como la tendencia general a nivel nacional (MECD, 2013). Situación similar a su vez, aunque algo más acuciado debido al tejido empresarial de Argentina, se puede encontrar en este país como manifiestan los estudiantes de la UCC. De hecho, informes como el realizado por Stumpo y Rivas (2013) para CEPAL de Naciones Unidas, demandan un incremento en Argentina de la movilidad de capital humano avanzado y establecimiento de plataformas tecnológicas de comunicación para avanzar con mayor rapidez en la integración de los mercados del trabajo internacionales.

Ante los ya conocidos cambios y transformaciones sociales y laborales, llama la atención el elevado porcentaje de estudiantes de la UM cuya intención es buscar cualquier tipo de trabajo al terminar sus estudios. Los fenómenos de sobrecualificación publicados recientemente en la literatura científica (Homs, 2014; Kucel y Vilalta-Bufí, 2013), empiezan a generalizarse en España con motivo del desajuste existente entre la oferta del sistema educativo y la demanda de trabajadores cualificados, sin olvidar la actual escasez de ofertas de puestos de trabajo. Por su parte, para los estudiantes de la UCC, la opción de dedicarse a su familia es una de las más repetidas, debido quizás a la mayor proporción de mujeres en la Universidad de Argentina estudiada, a las mayores limitaciones y discriminación laboral que viven actualmente éstas en el mercado laboral o bien, por motivos puramente culturales (Brunner y Ferrada Hurtado, 2011; M. Noriega, Maris y Maris, 2015).

A lo largo de este trabajo se destacan actitudes y expectativas diferentes según la universidad considerada al presentar necesidades heterogéneas y realidades encontradas. Se comprueba que el tránsito a la vida activa es un complejo proceso cargado de incertidumbre e inestabilidad socioeconómica, donde se combinan múltiples factores que condicionan y mediatizan los tres grandes vértices del triángulo formación-orientación-empleo, con independencia del país o universidad en la que se estudie. Todo un proceso donde se entrelazan situaciones y factores complejos que pueden actuar como obstaculizadores o facilitadores y cuyo éxito reside no tanto en la obtención de un empleo, sino de la realización resultante de acceder al mismo de acuerdo con las preferencias personales, sociales, profesionales y vitales.

Pese a las limitaciones de este estudio, donde la muestra se ha localizado en dos universidades y facultades determinadas y se dispone de la visión única del alumnado, este trabajo puede y deber entenderse como una interesante aportación empírica realizada en el primero de los tres momentos clave del proceso de transición a la vida activa, que habrá que completar con estudios posteriores que aborden a su vez, las aportaciones de docentes, empleadores, administraciones educativas y demás agentes sociales.

La educación superior es un proyecto social que no termina con la educación y la formación profesional. Necesita que los egresados, se aseguren una oportunidad para desempeñar su formación de forma productiva. Es decir, que se planifique y se potencie el proceso educativo para la transición de los egresados al mundo del trabajo, la transición a la vida activa, su inserción socio-laboral. 


\section{Referencias}

Alonso, L. E., Fernández Rodríguez, C. J. y Nyssen, J. M. (2009). El debate sobre las competencias: una investigación cualitativa en torno a la educación superior y el mercado de trabajo en España. Madrid: ANECA.

Bauman, Z. (2008). La vida líquida. Barcelona: Paidós.

Blossfeld, H. P. y Mills, M. (2010). How does globalization affect the life courses of young men and women in modern societies?. En J.C. Tremmel (Ed.), A young generation under pressure? (pp. 37-56.). Nueva York, EE.UU.: Springer.

Brunner, J. J. (2012). La idea de universidad en tiempos de masificación. Revista Iberoamericana de Educación Superior, 7(3), 130-143. Recuperado de http://www.redalyc. org/articulo.oa?id=299129031008

Brunner, J. J. y Ferrada Hurtado, R. (2011). Educación Superior en Iberoamérica. Informe 2011 (Informe No. 209.354). Santiago de Chile: Centro Interuniversitario de Desarrollo (CINDA).

Centeno Moreno, G. y Cubo Delgado, S. (2013). Evaluación de la competencia digital y las actitudes hacia las TIC del alumnado universitario. Revista de Investigación Educativa, 31(2), 517-536. Doi: 10.6018/rie.31.2.169271.

de la Hoz, F. J., Quejada, R. y Yánez, M. (2012). El desempleo juvenil: problema de efectos perpetuos. Revista Latinoamericana de Ciencias Sociales, Niñez y Juventud, 10(1), 427-439. Recuperado de http://www.scielo.org.co/pdf/rlcs/v10n1/v10n1a27

Dumitriu, C., Timofti, L. C. y Dumitriu, G. (2014). Communicative Skill and/or Communication Competence?. Procedia-Social and Behavioral Sciences, 141, 489-493. doi: 10.1016/j.sbspro.2014.05.085

Echevarría, B. (2008). Competencias y cualificaciones. En B. Echevarría (Coord.), Orientación profesional (pp. 69-124). Barcelona: UOC.

Escolano, C., García-Montoya, E., Pallàs, M., Miñarro, M., Vázquez, S., Marqués, A. y Lluch, A. (2015). Pasaporte a la profesión en el Grado de Farmacia. REIRE, Revista d'Innovació i Recerca en Educació, 8(1), 1-16. doi: 10.1344/ reire2015.8.1811

García de Fanelli, A. y Jacinto, C. (2010). Equidad y educación superior en América Latina: El papel de las carreras terciarias y universitarias. Revista Iberoamericana de Educación Superior, 1(1), 58-65. Recuperado de https://ries.universia.net/article/viewFile/33/92

García-Montalvo, J. y Peiró, J. M. (2012). Crisis económica e inserción laboral de los jóvenes: Resultado del Observatorio de Inserción Laboral de los Jóvenes 2011. doi: 10.12842/ OBSERVATORIO_2011

Hager, P. J. y Holland, S. (2006). Graduate attributes, learning and employability. Dordrecht, Países Bajos: Springer.

Homs, O. (2014). Garantía Juvenil en España, ¿Qué podemos aprender de experiencias internacionales?. Barcelona: Fundación Bertelsmann.

Isus, S. (2008). Concepto y funciones de la Orientación Profesional. En B. Echeverría (Coord.), Orientación profesional (pp. 125-172). Barcelona: UOC.

Jurado de los Santos, P., Olmos Rueda, P. y Pérez Romero, A. (2015). Los jóvenes en situación de vulnerabilidad y los programas formativos de transición al mundo del trabajo. Educar, 51(1), 211-224. Recuperado de http://www.raco.cat/index.php/ Educar/article/view/287042 
Kessler, G. (2010). La disyunción educación-trabajo en el Mercosur. Coincidencias y diferencias entre jóvenes de Argentina, Brasil, Paraguay y Uruguay. Propuesta Educativa, 34(2), 53-64. Recuperado de http://www.propuestaeducativa.flacso.org. ar/dossier_articulo.php?id=45\&num $=34$

Kucel, A. y Vilalta-Bufí, M. (2013). Job satisfaction of university graduates. Revista economía aplicada, 21(61), 29-55. Recuperado de http://www.redalyc.org/pdf/969/96925716002.pdf

Martínez Clares, P. (2008). Orientación profesional para la transición. En B. Echeverría (Coord.), Orientación profesional (pp. 223-300). Barcelona: UOC.

Matsaganis, M. Rabemiafara, N. y Ward, T. (2013). Young people and temporary employment in Europe. Recuperado del sitio de Internet de European Foundation for the Improvement of Living and Working Conditions, Catania, University of Catania: http://www.eurofound.europa.eu/sites/default/files/ef_publication/field_ef_document/ef1377en.pdf

Maurizio, R. (2011). Trayectorias laborales de los jóvenes en Argentina: ¿Dificultades en el mercado de trabajo o carrera laboral ascendente? (Serie Macroeconomía del Desarrollo, № 109). Santiago de Chile: Naciones Unidas - CEPAL.

Ministerio de Educación, Cultura y Deporte (2013). Datos básicos del sistema universitario español. Curso 2013-2014. Madrid: Autor.

Noriega, J. E. y Montiel, M. C. (2014). La universidad argentina entre sus regulaciones y tendencias. Revista Iberoamericana de Educación Superior, 5(12), 88-103. doi: 10.1016/ S2007-2872(14)71944-4

Noriega, M., Maris, S. y Maris, S. (2015). Deserción en estudiantes de nuevo ingreso a carreras de diseño. El caso de la Universidad de Buenos Aires, Argentina. Revista Electrónica Actualidades Investigativas en Educación, 15(1), 1-24. Doi: dx.doi. org/10.15517/aie.v15i1.17735.

Organización Internacional del Trabajo (2013). Tendencias Mundiales del Empleo Juvenil 201. Una generación en peligro. Ginebra: Autor.

Sabariego-Puig, M., Vilà-Baños, R. y Sandín-Esteban, M. P. (2014). El análisis cualitativo de datos con ATLAS.ti. REIRE. Revista d'Innovació i Recerca en Educació, 7(2), 119-133. doi:10.1344/reire2014.7.2728.

Stumpo, G. y Rivas, D. (Comp.) (2013). La industria argentina frente a los nuevos desafios y oportunidades del siglo XXI. Santiago: CEPAL. (LC/L.3637).

Vázquez García, J. A. (2015). Nuevos escenarios y tendencias universitarias. Revista de Investigación Educativa, 33(1), 13-26. doi:dx.doi.org/10.6018/rie.33.1.211501.

Watts, A. G. (2006). Career development learning and employability. En M. Yorke (Ed.), Learning and employability series. York, Reino Unido: Higher Education Academy.

Zabalza, M. A. (2011). El practicum en la formación universitaria: estado de la cuestión. Revista de Educación, 354, 21-43. Recuperado de http://www.revistaeducacion.mec. es/re354_02.html

Fecha de recepción: 10/07/2015

Fecha de revisión: 12/07/2015

Fecha de aceptación: 07/08/2015 
